Lorenz Böllinger

\title{
Grenzenloses symbolisches Strafrecht Zum Cannabis-Beschluß des Bundesverfassungsgerichts ${ }^{\mathrm{I}}$
}

\section{Kontext des Cannabis-Beschlusses}

Über drei Jahre hatte das Bundesverfassungsgericht gebraucht, um über den Vorlagebeschluß des LG Lübeck vom 19. 12.19912 zu entscheiden. Der Senat hat fünf weitere, äholich gelagerte und gleichfalls ausschließlich Cannabis betreffende Vorlagebeschlüsse nach Art. I00 GG sowie eine entsprechende Verfassungsbeschwerde einbezogen. ${ }^{3}$ Dabei ging es um zwei je hinsichtlich Konsum- oder Weitergabeorientierung polarisierbare Gruppen von Tatbestandsmerkmalen: Tathandlung Erwerb, Einfuhr, Besitz ( $\$ 29$ Abs. r u. 3 BtMG) vs. Abgabe, Handeltreiben ( $\$ 29$ Abs. $x$ )

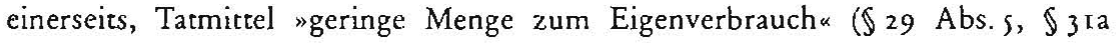
BtMG) vs. "nicht geringe Menge « ( $\$ 29$ Abs. 3 BtMG a. F.) andererseits. Bekanntlich wurden diese Tatbestände von der Mehrheit des Senats ( $7: 1$ ) rundweg für verfassungskonform erklärt. Eher beiläufig wurde lediglich eine einheitlichere und - unter bestimmten Voraussetzungen - obligatorische Anwendung der Opportunitätsnorm des $\S_{3}$ 1 a BtMG gefordert.

Gemessen an diesem Gehalt war das unmittelbare Echo auf den Cannabis-Beschluß des Bundesverfassungsgerichts v. 9.3. 1994 spektakulär und unverhältnismäßig: Von »Wende in der Drogenpolitik«, Dammbruch und Gefahr für die Jugend war die Rede, in der BILD-Zeitung sagten Prominente balkendick »Nein! «. Alsbald war die Drogenpolitik wieder, wenn auch nur vorübergehend, Wahlkampf-Thema, gab es Gesetzesinitiativen zur neuerlichen repressiven Novellierung des Betäubungsmittelgesetzes (BtMG). Schon diese verzerrte Wahrnehmung einer Entscheidung, die in Wirklichkeit hinter die geltende Gesetzeslage zurückgeht, erscheint mir interessant und interpretationswürdig. Das durch die Medien sofort angeheftete Etikett war öffentlich wirksamer als der veritable Tenor und Inhalt der Entscheidung. Daß die Medien die "Wende" plakarierten, mag einerseits darauf zurückzuführen sein, daß die Zeit wirklich »reif « erscheint für eine Umkehr in der Drogenpolitik. Es mag auch mit der marktgängigen Lust zu Dramarisierung und Hysterisierung zusammenhängen: wenn Wende, dann auch epidemische Ausbreitung der "Drogenseuche«. Ein weiterer Grund könnte sein, daß die weitläufige - wenn auch bundesweit äußerst diskrepante - Einstellungspraxis der Staatsanwaltschaften nach $\$ \mathbb{S}$ x $53 \mathrm{ff}$. StPO und die Praxis des gericht lichen Absehens von Strafe nach $\$ 29$ Abs. s BtMG bisher in der Öffentlichkeit kaum bekannt war, erst recht nicht die 1992 eingeführte Erweiterung der staatsanwaltschaftlichen Einstellungsmöglichkeiten nach $\$ 3$ ra BtMG hinsicht-

1 Ohne abweichende Meinungen abgedruckt in: StV 1994, 295 If. u. NJW r994, 1577 ff.; im folgenden wird nach dem Abdruck im SiV zitiert. Abw. Meinungen abgedr. in NJW 1994, is 8 sf. u. $1588 \mathrm{ff}$.

2 LG Lübeck NJW 1992, 1571 ff.

3 Sieben weitere Vorlagebeschlusse wurden niche zugelassen, darunter LG Frankfurt StV 1993, 77 ff., LG Hildesheim. DVJJ-Journal $1992,117 \mathrm{ff}$. 
lich konsumorientierten Umgangs mit geringen Mengen jeglicher illegaler Drogen, worüber sich CDU-Politiker auf einmal so entsetzt zeigten. Es mag auch - und das wäre die positivste Interpretation - mit Begründungsinhalten des Beschlusses zu tun haben, die $z$ war nicht entscheidungsrelevant geworden sind, aber gleichwohl aufhorchen lassen. So kommen Besprechungen des Beschlusses vereinzelt zu recht liberalen Deutungen, z. B.: das BVerfG habe die Schrittmacherfunktion des Betäubungsmittelstrafrechts erkannt (!) und dem Gesetzgeber im Sinne einer mzeitlichen und inhaltlichen Konditionierung * aufgegeben, das Konzept der »Trennung der Märktc" nach holländischem Muster zu testen. ${ }^{4}$ Die vagen obiter dicta der Entscheidung sind aber genauso gut in der repressiven Richtung interpretierbar.

Wie auch immer: die Sensation war auf dem Medienmarkt schnell vernutzt. Es stabilisiert sich seither der - erst noch empirisch zu belegende - Eindruck einer zunehmenden faktischen Entspannung der Strafverfolgung von Cannabis-Konsumenten, auch wenn die vom BVerfG angemahnten bundeseinheitlichen Richtlinien noch auf sich warten lassen und möglicherweise überhaupt nicht funktionieren können.' Außerdem scheint sich der Beschluß informell bereits hinsichtlich einer ohnehin überfälligen generellen Revision der betäubungsmittelrechtlichen Mengenbegriffe auszuwirken. ${ }^{6}$

Unabhängig von der relativen Offenheit für die zukünftige Interpretation und Novellierung dcs BtM-Rechts sehe ich die viel weitergehende und wegen seiner Irrevisihilität gravierende Bedeutung des Beschlusses in der Begründung. Durch sie werden elementare Prinzipien des rechtsstaatlich-liberalen Strafrechts angetastet und eine im doppelten Sinne grenzenlose Strafrechtlogik installiert: Die Auflösung der Limitierungsfunktion von Tatbestand, Rechtsgut und Schuld einerseits, und die bedingungslose Überlassung der "Definitionsmacht für gesetzliche Zielsetzungen und die Zwecktauglichkeit von Strafvorschriften $\alpha^{7}$ an internationale Gremien andererseits. Einen Lichtblick stellt lediglich die für Reformüberlegungen bedeutsame abweichende Meinung des Senatsmitgliedes Sommer dar. ${ }^{8}$ Eine weitere abweichende Meinung der Richterin Graßhof bezichtigt gar die Senatsmehrheit einer zu laschen Haltung.

\section{Kritik der Begründung}

Das Hauptgewicht des Beschlusses betrifft die Grundrechtsprüfung der allgemeinen Handlungsfreiheit (Art. 2 Abs. I GG) und des in Art. 2 Abs. 2 S. 2 GG verbrieften Freiheitsrechts. Nur kurz setzt sich das Gericht am Schluß auch mit Art. 2 Abs. 2 S. I und Art. 3 Abs. I GG auseinander. Vorweg ist anzumerken, daß die ausführliche strafrechtsdogmatische und verfassungsrechtliche Diskussion, welche zu diesen Fragen seit einiger Zeit in den Fachmedien geführt wird, vom BVerfG souverän übergangen wird.9

4 Vgl. Schneider, Hartmut: Haschisch im sanktionsfreien Raum - das Konsumverhalten im Lichte der Entscheidung des Bundesverfassungsgerichts, StV 1994, 390.

s Ausfuhrlich dazu Nelles, Ursula/Velten, Petra: Einstellungsvorschrifren als Korrektiv für unverhaltnismáBige Strafgesetze? Anm. z. Cannabis-Beschluß d. BVerfG, NJW 1994, 1577, in: NStZ 1994, $366 \mathrm{ff}$.

6 Vgl. dazu Bollinger, Lorenz/Stöver, Heino: Drogenpraxis, Drogenrecht, Drogenpolitik. 4. Auflage, Frankfurt 1994, S. $142 \mathrm{ff}$.

7 Nellesivelten (Fn. S), 366

8 NJW $1994,1588 \mathrm{ff}$.

9 Vgl. z. B. Hund, Horst: Von der Repression zur Legalisierung, ZRP 1993,213 ff.; Kaschkag, Hannes: Verfassungsrechtliche Grenzen strafrechtswirksamer Legislativtechnik. FS Krause, Köln etc. 1990, S. 123 ff.; Kòh- 
I. Hinsichtlich Art. 2 Abs. 1 GG wird zunächst postuliert, daß »der Umgang mit Drogen, insbesondere das Sichberauschen, aufgrund seiner vielfältigen sozialen Ausund Wechselwirkungen nicht " zum Kernbereich privater Lebensgestaltung gerechnet werden könne, welcher absolut geschützt und damit der Einwirkung der öffentlichen Gewalt entzogen sei. ${ }^{10}$ Die darin zum Ausdruck kommende empirische Prämisse wird nicht weiter begründet. Insbesondere wird übergangen, daß zumindest ein erheblicher Anteil der manifesten sozialen Auswirkungen kriminalisierungsbedingt ist. Auch rechtlich erliegt die Begründung einem Zirkelschluß: Die allgemeine Handlungsfreiheit sei nur in den Schranken des 2. Halbsatzes des Art. 2 Abs. I GG gewährleistet und stehe damit insbesondere unter dem Vorbehalt der verfassungsmäßigen Ordnung. Weil darunter alle formell und materiell mit der Verfassung in Einklang stehenden Rechtsnormen - also auch das BtMG - zu verstehen seien, verletze die Beschränkung der allgemeinen Handlungsfreiheit nicht Art. 2 Abs. 1 GG. Das war ja erst zu beweisen. Es ist weiter nicht begründet, warum Cannabis-Umgang und "Sichberauschen " nicht ebenso zum Kernbereich der allgemeinen Handlungsfreiheit gehören wie z. B. das Essen, die zwischenmenschliche Kommunikation, die sexuelle Betätigung oder das Hantieren mit Messern, welche wie alle denkbaren menschlichen Handlungen - unter bestimmten Umständen "soziale Aus- und Wechselwirkungen « haben und nur hinsichtlich solcher Umstände, nicht aber generell, spezifischen rechtlichen Beschränkungen ausgesetzt werden. Diese - im Justizalltag natürlich übliche - methodologische Unreflektiertheit des Vorgehens setzt sich in der weiter unten zu erörternden Rechtsguts-Novität »Gestaltung des sozialen Zusammenlebens « fort.

Positiv zu bewerten ist an dieser Passage, daß das Sichberauschen überhaupt als eine Form menschlichen Handelns anerkannt wird, welche in den - wenn auch der Schrankentrias unterfallenden - Bereich der allgemeinen Handlungsfreiheit gehört. Darin sehe ich einen ersten Ansatz zum Respekt vor der freien Entscheidung zu Drogenkonsum.

Zu begrüßen ist auch, daß in materieller Hinsicht allein auf das Verhältnismäßigkeitsprinzip als Maßstab abgestellt wird und wie dieses elementarste Verfassungsprinzip verfassungs- und strafrechtlich operationalisiert wird: ihm komme "gesteigerte Bedeutung für die Prüfung einer Strafvorschrift zu, die als schärfste dem Staat zur Verfügung stehende Sanktion ein sozialethisches Unwerturteil über ein bestimmtes Handeln des Bürgers ausspricht. « Nur »aus besonders gewichtigen Gründen « dürfe in das durch Art. 2 Abs. 2 S. 2 GG geschützte Grundrecht der Freiheit der Person eingegriffen werden, d. h. nur, "wenn der Schutz anderer oder der Allgemeinheit dies unter Berücksichtigung des Verhältnismäßigkeitsgrundsatzes erfordert. " " Besonders betont - und mit redundanten Selbstreferenzen des BVerfG belegt - wird die Voraussetzung einer Gesamtabwägung "zwischen der Schwere des Eingriffs und dem Gewicht sowie der Dringlichkeit der ihn rechtfertigenden Gründe« sowie die besondere Maßgabe des aus Art. I Abs. I GG abzuleitenden Schuldprinzips für eine "gerechte" Rechtsfolge.

\footnotetext{
ler, Michael: Irreiheitliches Rechtsprinzip und Betäubungsmittelstrafrccht, ZStW 1992, 3 ff.; ders.: Rechtsgut, Tatbestandsstruktur und Rechtswidrigkeitszusammenhang, MDR 1992, 739 ff.; Kreuzer, Arthur: Cannabisprohibition verfassungswidrig? Anm. zum Beschluß des Landgerichts Lubeck, in: Sucht 1992, $201 \mathrm{ff}$; ders.: Zur Bewertung von Haschisch in der Strafrechtssprechung des Bundesgerichtshofes, DRiZ 1991, $173 \mathrm{ff}$.; Messmer, Hermann: Haschischverbor und Gleichbehandlung. ZRP 1970, 80 ff.; Nestler-Tremel, Cornelius: Anm. 2u BGH StV 1992, 272; ebd. S. 273 ff.; Schneider, Hartmut: Anm. zu BGH StV 1992, 51 3; ebd., S. 514; ders. : Zur Entkriminalisierung der Konsumverhaltensweisen des Betäubungsmittelstraf rechts im Lichte internationalrechelicher Verpflichtungen, StV 1992, 489 ff.; Bollinger, Lorenz: Betäubungsmittelstrafrecht, Drogenpolitik und Verfassung, KJ 24/1991, S. $393 \mathrm{ff}$.

10 BVerfG StV 1994 , S. 296

it Ebd. S. 297.
} 
2. Soweit so gut. Die Durchführung dieser hehren, methodisch und inhaltlich jedoch abstrakten und unverbindlichen Grundsätze läßt aber stark zu wünschen übrig. Im weiteren Duktus des Beschlusses häufen sich Indizien für Oberflächlichkeit, mangelnden Bezug zur Wirklichkeit, Widersprüchlichkeit, insgesamt also mangelnde Sorgfalt im Hinblick auf die juristischen "Kunstregeln «. Es bestätigt sich der rechtssoziologische Befund, daß juristische Entscheidungen nicht deduktiv abgeleitet sind, sondern - sei es im Sinne einer willkürlichen normativen Setzung, sei es machtkommunikativ im Sinne eines politisch-personalen Aushandelungsprozesses im Scnat - induktiv »hergestellt» werden. Auch hier haben die empirischen Bezüge lediglich den Charakter nachfolgender Darstellung und vordergründiger Lcgitimation.

Der Senat vermengt zunächst in bedenklicher Weise zwei differenzierungsbedürftige Ebcnen: In einem zweiten Schritt nach Ausgrenzung des Cannabis-Umgangs aus dem Kernbereich der Handlungsfreiheit wäre zunächst am Maßstab des Verhältnismäßigkeitsgrundsatzes zu klären gewesen, ob und wie überhaupt eine rechtliche Beschränkung der allgemeinen Handlungsfreiheit i. S. der Schrankentrias notwendig ist und welche Optionen dafür in Betracht kämen. Erst im dritten Schritt hätte dann, cbenfalls an Hand des Verhältnismäßigkeitsgrundsatzes, die Verfassungskompatibilität der eingesetzten strafrechtlichen Mittel im Hinblick auf das Freiheitsrecht untersucht werden müssen. ${ }^{12}$ Statt dessen entwickelt der Senat den - m. E. sowohl verfassungsrechtlich wie strafrechtlich unhaltbaren - Kunstgriff einer doppelten Aufspaltung der Prüfung: Zwar wird der Verhältnismäßigkeitsgrundsatz schulmäßig operationalisiert in die Unter-Prinzipien Geeignetheit, Erforderlichkeit und Übermaßverbot. Diese werden dann jedoch in der Weise entkoppelt, daß die ersten beiden lediglich auf dic aus dem Gesamtzusammenhang der Strafbewehrung herausgelöste Komponente der Strafandrohung angewendet werden, der letzte dagegen auf die davon abgespaltene andere Komponente, nämlich Strafverhängung und -vollstrekkung. Das Verhältnismäßigkeitsprinzip ist aber unteilbar, ebensowenig wie das ultima-ratio-Prinzip der Kriminalpolitik. Zwar kann und muß die Strafe entsprechend dem Schuldpriazip und dem Verhältnismäßigkeitsprinzip abstufbar sein. $\mathrm{Ob}$ überhaupt dieses äußerste Mittel der Sozialpolitik, dieses gravierendste sozialethische Unwerturteil eingesetzt wird, muß aber nach den strafrechtstheoretischen Kriterien im Gesetzgebungsprozeß bestimmt worden sein. ${ }^{3}$

Von diesem Fehlgriff abgesehen ist nichts gegen die Auslegung durch den Senat einzuwenden, daß dem Gesetzgeber hinsichtlich »der Beurteilung der Eignung und Erforderlichkeit des gewählten Mittels zur Erreichung der erstrebten Ziele sowie bei der in diesem Zusammenhang vorzunehmenden Einschätzung und Prognose der dem Einzelnen oder der Allgemeinheit drohenden Gefahren « ein Beurteilungsspielraum zuzugestchen ist, welcher vom BVerfG nur begrenzt überprüft werden kann. ${ }^{14}$ Allerdings wäre es wichtig gewesen, hier über den Begriff der "Sachgerechtheit « der erforderlichen Abstimmung von Tatbestand und Rechtsfolge hinaus genauere Maßstäbe hinsichtlich der Rationalität und Schlüssigkeit der vom Gesetzgeber zugrundegelegten empirischen Annahmen zu formulieren. Denn es werden ja auch vom Gesetzgeber diesseits der Normierung, insofern der Wahrheitsfindung im Strafprozeß vergleichbar, Annahmen über die soziale Wirklichkeit gemacht. Es gilt das Prinzip »in dubio pro libertate«. Der gerade im strafprozessualen Revisionsrecht immer mehr hervorgehobene Anspruch an Rationalität und Widerspruchsfreiheit

12 So auch die abweıchende Meinung Sommer.

$13 \mathrm{Vgl}$. Hassemer in AK-StGB, Bd. 1, 1990, Vor $\oint_{1}$, Anm. ${ }_{183} \mathrm{ff}$.

14 BVerfG StV 1994, S. 297. 
justizieller Wirklichkcitserfassung sollte erst recht für den Gesetzgeber Maßstab sein. ${ }^{15}$

Besonders bedenklich ist die verfassungsgerichtliche Würdigung des Schutzgutes, ist doch die Rechtsgutsbestimmung das zentrale methodische Instrument sowohl zur Begründung als auch zur Begrenzung des staatlichen Strafanspruches. ${ }^{16}$ Das verfassungs- und strafrechtliche Schutzgut wird vom Senat unter Bezugnahme auf die Gesetzgebungsmaterialien geprüft: Aufgabe des BtMG sei es, „die menschliche Gesundheit sowohl des Einzelnen wie der Bevölkerung im ganzen vor den von Betäubungsmitteln ausgehenden Gefahren zu schützen und dic Bcvölkerung, vor allen Jugendliche, vor Abhängigkeit von Betäubungsmitteln zu bewahren $* .{ }^{17} \mathrm{Zur}$ Erreichung dieses Zweckes stelle "der Gesetzgeber nicht nur Verhaltensweisen unter Strafe, die unmittelbar für die Gesundheit Einzelner gefährlich sind. Vielmehr geht es um die Gestaltung des sozialen Zusammenlebens in einer Weise, die es von sozialschädlichen Wirkungen des Umgangs mit Drogen freihält, wie sie auch von der sog. weichen Droge Cannabis ausgehen: Durch sie werden insbesondere Jugendliche an Rauschmittel herangeführt; ihre Gewöhnung an berauschende Mittel wird gefördert. Die Festigung der Persönlichkeit von Jugendlichen und Heranwachsenden kann behindert werden. «Darüber hinaus übernimmt das Gericht unhinterfragt die im Wiener Suchtstoff-Abkommen der UNO von 1988 enthaltenen, noch weitergehenden Annahmen über einen linearen Ursachenzusammenhang zwischen Drogenkonsum, -verkehr und den Gefahren sowohl für "Gesundheit und Wohl der Menschen « als auch für »die wirtschaftlichen, kulturellen und politischen Grundlagen der Gesellschaft « überhaupt. Das BtMG sei der deutsche Beitrag zur internationalen Kontrolle dieser Gefahren. Mit diesen Zielsetzungen diene das BtMG Gemeinschaftsbelangen, welche vor der Verfassung Bestand hätten.

Damit ist die wegen ihrer Vagheit und Diffusität sowie ihrer totalitären Konnotation, vor allem aber wegen des wissenschaftlichen Nachweises der medizinischen Unschädlichkeit des Tetrahydrocannabinol (THC) zunehmend angefochtene Rechtsgutsdefinition "Volksgesundheit" des BtMG von 1971 nachträglich enorm aufgerüstet worden. ${ }^{18}$ Diesen Weg hatte im Urteil vom 25. 8. 1992 bereits der BGH gebahnt, durchaus mit Blick auf das beim BVerfG schwebende Vorlageverfahren ${ }^{19}$ : vom Schutz vor »drogenbedingten Schwierigkeiten und Leistungsabfällen in Ausbildung, Beruf und Familie « ist da die Rede.

Damit wirft das BVerfG den bislang allseits konsentierten, elementaren personalen Rechtsgutsbegriff über Bord, wonach auch die Definition sozialer, kollektiver Rechtsgüter auf ein Substrat konkret-individualisierbarer potentieller Opfer zurïckgeführt werden können muß. ${ }^{20}$ Selbstschädigung darf aus Gründen des Art. 2 Abs. I GG nicht Gegenstand strafrechtlicher Intervention sein. Bei den von BGH und BVerfG definierten Objekten der Drogengefahr (Beruf, Familie etc.) handelt es sich aber um Abstrakta, um soziale Wertkonstrukte, welche nicht mehr im Sinne eines "relevanten Anderen « (G. H. Mead) konkretisierbar und individualisierbar sind. Der weiteren Ausweitung von Rechtsgutsdefinitionen sind damit die Grenzen entzogen. Jegliche Verletzung von staatlich definierten Interessen kann so zur Rechtsgutsverletzung erklärt werden, welche mit Strafe zu bewehren sei. Beispielsweise könnte,

is Vgl. Albrechr, Peter-Alexis: Ubberzeugungsbildung und Sachverständigenbeweis in der neueren strafrechtlichen Judıkatur zur freien Beweiswiirdigung ( $\$ 261$ StPO), NStZ 1983, $486 \mathrm{ff}$

16 Vgl. die umfassende Darstellung bei Hassemer, AK-StGB, Bd. I, 1990, Vor $\$$ r, Anm. $183 \mathrm{ff}, 255 \mathrm{ff}$. m.w.N.

17 BVerfG NJW 1994, 1579 .

18 So auch Schneider 1994, (Fn. 4), 390 f.

19 BGH StV 1992, S. S13.

$20 \mathrm{Vgl}$. Hassemer, (Fn. 16), Anm. $28 \mathrm{gf}$. 
wegen seiner gesundheitsschädlichen Auswirkungen und der sozialgefährlichen triebhaften Komponente, der Konsum von Zucker unter Strafe gestellt werden.

Noch abstrakter und diffuser ist die gleichfalls entwickelte Konstruktion eines notwendigen Schutzes der internationalen Völkergemeinschaft und der internationalen Beziehungen der Bundesrepublik. Wenn der Drogentäter auch dafür in Anspruch genommen wird, fehlt zudem der erforderliche Schutzzweckzusammenhang der Strafnorm und ist Art. r Abs. I GG verletzt, weil er zum Objekt anderweitiger staatlicher Interessen gemacht wird. »Das dem Strafrecht immanente Kausalitäts-, Zurechnungs- und Schädigungsprinzip ist de facto aufgelöst. " ${ }^{2 t}$ Nach noch geltender Verfassung darf sich der Gesetzgeber die Rechtsgutsdefinition nicht ohne weiteres von supranationalen Instanzen vorgeben lassen. Es besteht auch im Hinblick auf die verschiedenen UNO-Konventionen keine absolute völkerrechtliche Verpflichtung zu einem Totalverbot oder gar zur Pönalisierung von Cannabis. ${ }^{22}$

Im übrigen wird durch solche Rechtsguts-Diffusion die allgemein feststellbare Vorverlagerung und Umwidmung des Strafrechts zu einem "Verbrechens-Vorsorgerecht «, also zu einem Polizeistrafrecht weiter vorangetrieben. ${ }^{23}$ Es erscheint alarmierend, daß der Senat offenbar keinerlei grundsätzliche strafrechtstheoretische Überlegungen angestellt hat und die entsprechende wissenschaftliche Diskussion ignoriert.

3. Die bis dahin im gewohnten Urteilsstil gehaltenen Feststellungen werden dann im Sinne einer Beweiswürdigung durch eine scheinbar auf nachbarwissenschaftliche Erkenntnisse bezugnehmende Diskussion der gesetzgeberischen Schadensbehauptungen unterfüttert. An deren Ende steht jedoch der Befund, daß wauch nach dem jetzigen Erkenntnisstand nicht unbeträchtliche Gefahren und Risiken « verbleiben, »so daß die Gesamtkonzeption des Gesetzes in bezug auf Cannabisprodukte auch weiterhin vor der Verfassung Bestand hat. ${ }^{24}$ Dieser Befund wird abschließend mit den "fachbehördlichen Stellungnahmen des Bundesgesundheitsamtes (BGA) und des Bundeskriminalamtes (BKA) sowie das vom Senat - über die zitierten zusammenfassenden Darstellungen hinaus - ausgewertete einschlägige Schrifttum « belegt.

Das methodische Vorgehen bei dieser "Beweiswürdigung « muß man sich etwas näher anschauen. Bei der genannten fachbehördlichen »Stellungnahme des BKA zu den kriminalistischen Auswirkungen des Konsums von Cannabis« handelt es sich um einen unveröffentlichten Bericht an den Bundesinnenminister aus dem Jahre 1992, der wegen seiner methodischen Schlampigkeit und inhaltlichen Fehlerhaftigkeit einer eigenen Kommentierung wert wäre. Beispielsweise werden darin 6 Suizidfälle des Jahres 1991 "eindeutig dem Cannabiskonsum « zugerechnet (S. Is des Berichts). Die Stellungnahme des BGA ist ein oberflächliches Resüme der spärlichen Cannabis-Empirie in Deutschland. Welches "einschlägige Schriftum« der Senat noch ausgewertet haben will, bleibt im Dunkeln. Bei den ansonsten angeblich verarbeiteten, jedenfalls aber zitierten zusammenfassenden Darstellungen handelt es sich um Repräsentanzen dessen, was man in Politik und Wissenschaft als "verschiedene Lager « bezeichnen kann: Täschner-Geschwinde-Quensel. ${ }^{25}$ Nach dem üblichen juristischen Muster setzt man sich mit nachbarwissenschaftlichen Erkenntnissen nicht

21 Schneider, (Fn. 4), S. 390.

22 Ausführlich dazu: Schneider, Hartmut: Zur Entkriminalisierung der Konsumverhaltensweisen des Betaubungsmittelstrafreches im Lichte internationaler Verpflichtungen. StV 1992, 489 ff.

23 Vgl. ebd. S. 391 ,

24 BVerfG SiV 1994, S. 297 f.

2s Taschner, Karl L.: Das Cannabisproblem, 3. Aufl., Wiesbaden 1986; Quensel, Stephan: Wirkungen und Risiken des Cannabisgebrauchs. In: Scherer, S./Vogt, I. (Hrsg.): Drogen und Drogenpolitik, Frankfur 1989, S. 379 ff.; Geschwinde: Rauschdrogen, 2. Aufl. 1990. 
etwa inhaltlich auseinander, was eine genuin wissenschaftliche Rczeption und Diskussion dieser und weiterer Literatur erfordern würde. Sondern man wägt auf einer vordergründigen Plausibilitätsfassade ab: Die eine "konservative " Position wird, scheinbar fair und gerecht, ebenso crwähnt, aber angezweifelt wie die andere, "progressive«. Also muß die "Wahrheit in der Mitte liegen, nämlich bei dem Juristen Geschwinde, der eine gemäßigt konservative Linie vertritt. Nicht umsonst wird Verfassungsrecht auch als Abwägungsrecht bezeichnet.

Positiv hervorzuheben - weil nicht mehr selbstverständlich - ist an dieser »PseudoDiskussion « allerdings die offizielle handlungstheoretische Maxime, daß rechtsstaatliche Gesetzgebung und Justiz sich überhaupt an Erkenntnissen der Nachbarwissenschaften zu orientieren haben. An anderer Stelle im Beschluß ${ }^{26}$ wird dicsbezüglich und glcichsam als Trostpflästerchen am Schluß - auch noch der in stetiger Rechtsprechung gefestigte Grundsatz betont, der Gesetzgeber habe eine Beobachtungs- und Überprüfungspflicht im Hinblick auf die »offene kriminalpolitische und wissenschaftliche Diskussion über die vom Cannabiskonsum ausgehenden Gefahren und den richtigen Weg ihrer Bekämpfung “. Dabei müßten auch die Erfahrungen des Auslandes und insbesondere die niederländische Strategie einer "Trennung der Drogenmärkte durch die Frcigabe von Cannabis überprüft werden. Auch wenn dieses Postulat eines Realitätsbezugs der Jurisprudenz in der Realität der Gesctzgebung ebenso wie im vorliegenden Beschluß nur Alibi und legitimatorischer Schein bleibt, eröffnet es doch einmal mehr die Gelegenheit, Gesetzgeber und Justiz ideologiekritisch am Portepce zu packen.

In solch resignativer Perspektive erscheint es auf der Ebene der konkreten Wertungen gleichfalls positiv, daß überhaupt der Schadensmythos angekratzt wird: Daß die Konsumentenzahl auf bis zu 4 Mio. geschätzt wird, relativiert per se das Verdikt von der Kulturfremdheit; daß 56,7\% seltenen Gelegenheitskonsum betreiben, entdramatisiert ebenso wie die Einsicht in die Mitbedingtheit der Cannabis-W/irkung durch "Set" und „Setting sowie die Vcrneinung von Toleranzbildung und körperlicher Abhängigkeit sowie einer "Schrittmacherfunktion« und des berüchtigten "amotivationalen Syndroms .

Gleichwohl bleibt dic Wahrnehmung und Verarbeitung der Sachfragen durch das BVerfG, gemessen am Reichtum der wissenschaftlichen Literatur über Cannabis, ein komplexitätsreduzierter Abklatsch, ja ein Zerrbild der Realität. Der Begriff der "Gesundheit des Einzelnen und der Bevölkerung - nur mühsam ist der faschistoide Begriff der "Volksgesundheit « vermieden und verbrämt - wird in keiner Weise geklärt oder operationalisiert. Im Widerspruch zu allen wiedergegebenen Belegen gegen die Annahme von Gesundheitsschäden durch Cannabis wird immer noch konstatiert, es seien »die unmittelbaren gesundheitlichen Schäden bei mäßigem Genuß als eher gering anzusehen ${ }^{27}$ Konträr dazu wird dann wiederum behauptet, Cannabiskonsum beinhalte die Möglichkeit einer - nicht weiter operationalisierten psychischen Abhängigkeit, und »der Dauerkonsum von Cannabisprodukten (könne) zu Verhaltensstörungen, Lethargie, Glcichgültigkeit, Angstgefühlen, Realitätsverlust und Depressionen führen « und dies vermöge "gerade die Persönlichkeitsentwicklung von Jugendlichen nachhaltig zu stören « - womit implizit doch wieder das »amotivationale Syndrom (AMS) « Urstände feiert. Auch der Einstiegsmythos wird durch die Hintertür der Annahme einer "Einheitlichkeit des Drogenmarktes « wieder eingeführt, ohne daß die Kriminalisierungsbedingtheit solcher "Einheitlichkeit" gesehen würde. Auch der aus dem erwähnten BKA-Bericht bezogene Kausal- 
nexus von Cannabis-Konsum und Straßenverkehrsunfällen ist wissenschaftlich so nicht haltbar: In den zum Beleg angeführten Fällen war ausnahmslos Alkohol in einer tatsächlich problematischen Wechselwirkung mit Cannabis an dem Bedingungsgefüge beteiligr ${ }^{28}$

Neben diesen immanenten Widersprüchen in der Auswertung des vom Senat erhobenen Materials ist vor allem \%u beklagen, daß der heutige Forschungsstand nicht im entferntesten berücksichtigt ist. Weder sind die vielfältigen Widerlegungen der Schadensbehauptungen noch ist die methodologische Kritik daran erfaßt worden: ${ }^{29} \mathrm{Bei}$ aufgeklärtem, genußorientiertem und ins Alltagsleben integriertem Gebrauch hat Cannabis keine Schäden zur Folgc. Mißbrauch im Sinne von hochdosiertem Dauerkonsum und fchlender soziobiografischer Integration ist äußerst selten und selbst dann in seiner Schadenswirkung (einzig durch das Rauchen des Cannabiskrautes, nicht durch den Wirkstoff THC an sich) weit entfernt von auch nur moderatem Nikotinkonsum. Wenn negative psychische Auswirkungen z. B. von hochdosiertem Dauerkonsum berichtet werden (z. B. das oben erwähnte "Amotivationale Syndrom"), sind diese cbenso wie der Dauerkonsum selbst nur als parallele und interaktive Resultatc vorgängiger Bedingungen und Verläufe adäquat verstehbar, nicht aber im Sinne primärer und statisch-linearer Kausalität erklärbar.

Unreflektiert und implizit wird die empirische Annahme ins Zentrum der Wertung gerückt, der Konsum wirke ansteckend. Diese Infektions-Theorie stammt von medizinischen Denkmodellen über Virus- und Bakterienkrankheiten und ist aus psychologischer Sicht unhaltbar: Menschliche Motivationsprozesse sind weitaus komplizierter als das die krude-deterministische Theorie nahelegt, wonach das schlichte Ansichtigwerden eines Cannabiskonsumenten oder die Weitcrgabe einer Drogenmenge das Handeln anderer Menschen in relevanten Ausmaß beeinflussen könne.

Schließlich ignoriert die Entscheidung in unverständlicher Weise die gesamten kriminologischen Erkenntnisse übcr die schädigenden und zirkulären Auswirkungen der Pönalisierung und Strafverfolgung. ${ }^{3 \circ}$ Nur am Rande sei vermerkt, daß - worauf noch zurückzukommen ist - der unstreitige medizinisch-pharmakologische Nutzen von Cannabis natürlich uncrwähnt bleibt.

4. Auch im folgenden Teil ( $\left.\mathrm{Cl}_{3}\right)^{31}$ erscheinen mir die Kunstregeln der verfassungsrechtlichen Dogmatik und Strafrechtstheorie nicht eingehalten: Selbst wenn man die - gerade widerlegten - Schadensbehauptungen einmal unterstellt, so liegt doch ein eklatanter normlogischer Fehler in der Zurechnung der Verantwortung dafür. Unsere Verfassungsordnung einschließlich des Strafrechts liegt, wie der allgemeinen Ethik, das Menschenbild des eigenverantwortlichen, selbstbestimmten und willensfreien Menschen zugrunde. Das impliziert die Annahme, der durchschnittliche Mensch sei in der Lage, sich selbst zu steuern. Dieses Menschenbild wird durch die krude und realitätsferne empirische Annahme desavouiert, bei Drogenkonsum funktioniere diese Selbststeuerung nicht, das Individuum sei - ohne im strafrechtlichen Sinne einsichts- und steuerungsunfähig, mithin schuldunfähig zu sein - nicht mehr

$28 \mathrm{Vgl.} \mathrm{auch} \mathrm{die} \mathrm{inzwischen} \mathrm{publizierte,} \mathrm{im} \mathrm{Auftrag} \mathrm{des} \mathrm{National} \mathrm{Institute} \mathrm{of} \mathrm{Drug} \mathrm{Abuse} \mathrm{(USA)} \mathrm{in} \mathrm{Maas-}$ trich/Holland durchgefuhrte und außerst grundliche Untersuchung von Robbe, Hindrik Jan: Influence of Marihuana on Driving. University of Limburg, Maastricht 1993.

29 Siehe die vom Senat offenbar nicht wahrgennmmene umfassende Verarbeitung der internationalen einschlägigen Literarur sowie deren Nachweis bei Quensel, (Fn. 25); s. a. Kreuzer, Arthur: Cannabisprohibition verfassungswidrig? Anm, zum Beschluß des Landgeriches Lübeck. In: Sucht 1992, 20rff., m.w.N.

30 Vgl. dazu Kreuzer, Arthur: Jugend, Drogen, Krimiralität, 3. Aufl, Neuwied/Darmstadt r987 sowic Quensel, (Fn. 25), S. 394 ni.w.N

31 BVerfG SrV 1994, S. 298 
willensfrei. ${ }^{32}$ Normativ wird ein Durchgriff vom Konsumenten auf den unbeteiligten Bürger in der Weise konstruiert, daß letzterer von der Vorbildwirkung des ersteren überwältigt und angesteckt werden kann, wie von Bakterien und Viren. Es geht also wohlgemerkt nicht um eine Art von direkter Schädigung fremder Rechtsgüter, wie man sie durch die Ansteckung mit Schnupfen-Viren (sozialadäquat!) oder mit HIVViren (strafbar!) bejahen könnte. Sondern es muß, um zu der behaupteten Risikoverwirklichung zu gelangen, ein Willensentschluß und eine Handlung des Opfers hinzutreten. Unausgesprochen, wirklichkeitsfern und im Gcgensatz zu vielen anderen "schlechten Vorbildern « wird also "das Opfer « in diesem besonderen normativen Kontext als nicht mehr willensfrei konstruiert.

Auch der nächste Wertungsschritt, nämlich die Behauptung, das Strafrecht sei "geeignet, die Verbreitung der Droge in der Gcscllschaft einzuschränken und die dadurch von ihr ausgehenden Gefahren im ganzen zu verringern «, wird vom Senat durch nichts hergeleitet oder belegt. Wiederum wird die umfangreiche kriminologische Erkenntnislage und kriminalpolitisch-wissenschaftliche Problematisierung der unbeabsichtigten, kontraproduktiven, ja destruktiven Neben- und Folgewirkungen der Kriminalisierung ignoriert. Dies läßt sich kaum anders deuten denn als selbstherrliche Apodiktik eines Spruchkörpers, der sich frei von übergeordneter Kontrolle weiß. Solches Selbstmißverständnis des BVerfG als geistig-moralische Steuerungsinstanz in der politischen Auseinandersetzung mit unerwünschten Kulturausprägungen und Lebensstilen ist uns nicht zuletzt im Abtreibungs-Urteil begegnet.

Gleichermaßen basislos ist die folgende Feststellung der Vertretbarkeit der Einschätzung des Gesetzgebers, „die strafbewehrten Verbote gegen den unerlaubtcn Umgang mit Cannabis seien auch erforderlich «. ${ }^{33} \mathrm{Zu}$ begrüßen ist zwar, daß sich das BVerfG nieht mit der inhaltlichen Letztentscheidung an die Stelle des Gesetzgebers begeben will und ausdrücklich als Grundsatz hervorhebt, daß »kriminologische Erkenntnisse im Rahmen der Normenkontrolle insoweit Beachtung erfordern, als sie geeignct sind, den Gesetzgeber zu einer bestimmten Behandlung einer von Verfassungs wegen gesetzlich zu regelnden Frage zu zwingen «. ${ }^{34}$ Daraus folgt aber die Notwendigkeit, im Rahmen der Normenkontrolle zu überprüfen, ob sich der Gesetzgeber inhaltlich mit der wissenschaftlichen Debatte um materiell-rechtliche Entkriminalisierung, um Alternativen zur Pönalisierung, um Konzepte ordnungs- und verwaltungsrechtlicher Regulierung und außerrechtlicher Prävention (z. B. durch Aufklärungspolitik analog der AIDS-Politik) auseinandergesetzt hat. Eine solche Überprüfung der Einhaltung der gesetzgeberischen Sorgfaltspflichten ist der Senat schuldig geblieben. Es wird lediglich die - von U.S.-amerikanischen Interessen dominierte und ihrerseits gegen wissenschaftliche Erkenntnis immunisicrtc - Einschätzung der UNO-Suchtstoffabkommen insoweit unhinterfragt übernommen, als "die Richtigkeit « des Einsatzes »strafrechtlicher Mittel« jedenfalls noch nicht "zwingend « widerlegt sei.

Hinsichtlich des dritten Auslegungsmaßstabs des Verhältnismäßigkeitsprinzips, nämlich des *Übermaßverbots $\alpha$, unterscheidet der Senat zwischen dem "grundsätzlichen Verbot des Umgangs mit Cannabisprodukten und sciner Bewehrung durch die Androhung von Kriminalstrafe für die verschiedenartigen Verstöße gegen das Verbot*. Wie bereits erwähnt ist ein solches Auseinanderdividieren sowohl des Verhältnismäßigkeitsprinzips als auch des Strafrechts unhaltbar. Schon die Androhung von Strafe bewirkt wegen der Eröffnung der Möglichkeit von Strafverfolgungsmaß-

32 Ausführlich dazu Köhler, (Fn. 9), S. 3 ff.

33 BVerfG StV 1994, S. 298 am Ende.

34 Ebd. S. 299. 
nahmen einen Eingriff nicht nur in die allgemeine Handlungsfreiheit (Art. 2 Abs. I GG), sondern auch in das Freiheitsrecht des Art. 2 Abs. 2 S. 2 GG. 35

Die Rechtfertigung des Totalverbots durch "wichtige Gemeinschaftsbelange ist sowohl inhaltlich wie methodologisch unzulänglich. Zwar sind zwei der drei inhaltlichen Begründungsstränge folgerichtig: Soweit das Gericht zuvor zum Ergebnis "nicht unbcträchtlicher Gefahren und Risiken" für die Gesundheit der Bevölkerung und insbesondere der Jugend gekommen war, hatte es immerhin gemeint, daß sich diese waus heutiger Sicht als geringer darstellen, als der Gesetzgeber bei Erlaß des Gesetzes angenommen hat «. Daraus ergibt sich aber dic Notwendigkeit einer fundierten Begründung, warum bei solch veränderter Sichtweise die Angemessenheit der Grundrechtseinschränkung nach wie vor zu bejahen sei. Insoweit fehlt es auch hier an der erforderlichen Differenziertheit und Gründlichkeit der Abwägung: Wesentliche empirische und rechtswissenschaftliche Aspekte werden nicht, wie es eine differenziertc Dogmatik erfordert, diskutiert und bewertet. Zumindest hätte das Gericht in Anwendung seiner eigenen Grundsätze betreffend die gesetzgeberische Beobachtungs- und Übcrprüfungspflicht eine diesbezügliche Nachlässigkeit des Gesetzgebers rügen müssen. In übrigen imponiert die Unverfrorenheit des Zirkelschlusses, daß die Bevölkerung auch vor "den krimincllen Organisationen, die den Drogenmarkt beherrschen, und ihrem gemeinschädigenden Wirken « geschützt werden müsse: Ist es doch gerade das strafrechtliche Verbot, wclches diese Gefahr erst heraufbeschwört. ${ }^{36}$

Die Strafbewehrung des Totalverbots als das schärfste Mittel der Sozialkontrolle wird vom Senat einmal mehr mit der Wichtigkeit der zu schützenden Gemeinschaftsbelange legitimiert. Dabei fehlt es, wie schon im Gesetzgebungsprozeß r970 auf allen Ebenen, der Gesetzgebungs- und Strafrechtstheorie sowie der Dogmatik an Kriterien und Maßstäben dafür, was denn als "wichtig « anzusehen bzw. wo dic Schwelle der Strafbewehrung anzusetzen sei. Wie schon der Gesetzgeber erspart sich das BVerfG die Analyse solcher Gemeinschaftsbelange auf der empirischen Ebene. Dementsprechend wird auch das gesetzgeberische Versäumnis, sich ein Bild der sozialen Wirklichkeit zu verschaffen, nicht moniert. So hätte z.B. der Versuch eines empirisch begründeten Vergleichs mit anderen typischen Gcfahrenlagen in der Gesellschaft unternommen werden können.

s. In diesem Zusammenhang wird auch die ausdifferenzierte strafrechtswissenschaftliche Dogmatik der abstrakten Gefährdungsdelikte nicht berücksichtigt, wonach dieses kriminalpolitische Instrument wegen seiner tendenziell grenzenlosen Vorverlagerungswirkung nur restriktiv und in Relation zur Gewichtigkeit des zu schützenden Rechtsguts einzusetzen sei. Das Drogenstrafrecht bleibt denn auch nunmehr mit dem Segen des BVerfG - Schrittmacher für die ausufernde Nutzung dieses - wirkliche und wirksame Sozialpolitik ersetzenden - Instruments. Dies geschieht durch eine faktische Umkehrung des Regel-Ausnahmeverhältnisses: Trotz des auch vom BVerfG geschenen, rechtstatsächlich-emipirisch evidenten Überwiegens von Begehungsformen, die - bei immanenter Betrachtung - von geringer Gefährlichkeit für die geschützten Rechtsgüter sind, wird aus "generalpräventiven Gesichtspunkten « von den -- empirisch seltenen - gefährlicheren Tatvarianten, welche ein hohes $\mathrm{Maß}$ an sindividuellem Unrechts- und Schuldgehalt indizieren, als

35 So auch die abweichende Meinung von Sommer.

36 Ebd.; hier wird man an dic Auffangposiuion erinnert, welche dic Beibchaltung des $\$ 175$ StGB jahrelang zirkulär légitimierte: $Z_{w a r}$ sei Homosexualität nicht an sich schädlich, aber die ausgrenzenden und dissozialisierenden Einflüsse, denen die Homosexuelten ausgeserze seien - und welche maßgeblich von der Kriminalisierung ausgingen - müßten den Jugendlichen mic Hilfe des Stralrechts erspart werden. 
Regelfall ausgegangen. ${ }^{37}$ Dabei geht es insbesondere um "Handeltreiben " und "Abgabe« ( $\$ 29$ Abs. I Nr. I BtMG). Nur bei den konsumbezogenen Tatmodalitäten "Erwerb" (Nr. I) und "Besitz" (Nr. 3) soll trotz der vom Senat zugeschriebenen Fremdgefährdung qua »Möglichkeit einer unkontrollierten Weitergabe der Droge an Dritte ausnahmsweise, »je nach den Eigenschaften und Wirkungen der Droge, der im Einzelfall betroffenen Menge, der Art des jeweils in Betracht kommenden Verstoßes sowie unter Berücksichtigung sonstiger gefahrrelevanter Umstände « eine geringere Gewichtung durch den Einzelfallrichter möglich sein, wcil sonst das Übermaßverbot verletzt sein könnte.

Strafrechtstheoretisch ist intcressant, daß hier eine weitere Abstraktionsebene eingeführt wird: Die Cannabis-Droge wird, folgerichtig im Hinblick auf die vom Senat bejahten Schadensbehauptungen, abstrakt-generell als gefährlich für die geschützten, ihrerseits hochabstrakten Rechtsgüter angesehen. Die nächste Abstraktionsstufe liegt sodann - in Form des bereits angesprochenen logischen Hiatus - in der unausgesprochenen empirischen Unterstellung, daß die Weitergabe andere zum Konsum motivierc und dadurch den behaupteten Schaden realisiere, sowie in der normativen Zurechnung der Verursachung dieser Motivation. Eine noch weitergehendere Abstraktionsstufe ist mit der generellen Unterstellung erreicht, daß bei bewußt lediglich auf Eigenkonsum abzielendem Erwerb und Besitz ein Weitergabewille potentiell noch entstehen kann. Bewußte Weitergabeintentionen wären ja gemäß der Vorverlagerungs-Logik des BtM-Strafrechts als vollendetes, zumindest aber versuchtes $\mathrm{Han}$ deltreiben bzw. entsprechend als Abgabe zu subsumieren, wo bei gleicher Sachlage ansonsten im StGB eine straflose Vorbereitungshandlung oder allenfalls ein Versuch subsumiert werden könnte. Somit haben wir hier den einzigartigen Fall einer Vorverlagerung von Strafbarkeit in den Bereichen noch-nicht-bewußter, also unbewußter Handlungspotentiale und -risiken. Das ist mehr als Gesinnungsstrafrecht, das ist Psycho-Strafrecht!

Konkret-instrumentell wird der Drogenkonsum durch dic Dogmatik des BVerfG im übrigen auch noch zum Objekt im Sinne generalpräventiver $Z_{\text {wecksetzungen ge- }}$ macht: Gerade im Erwerb zum Eigenverbrauch verwirkliche sich die Nachfrage nach der Droge, "die den illegalen Drogenmarkt von der Nachfrageseite her konstituiert«, was bei geschätzten 800000 bis 4 Millionen Konsumenten nicht als unerheblich abgetan werden könne. ${ }^{38}$ Darin liegt eine weitere skurril anmutende Argumentationsfigur und Abstraktionsstufe, dic ihresglcichen im Strafrecht sucht: Die Einzelhandlung gilt nicht direkt und nicht für sich allein genommen als rechtsgutsgefährdend, sondern mittelbar über die Beflügelung des illegalen Marktes und nur im kumulativen Zusammenwirken mit Hunderttausenden von Gleichgesinnten. Diese dogmatische Figur mehrfach vermittelter bzw. abstrahierter Rechtsgutsgefährdung geht weit über den entsprechenden Gedanken im Umweltstrafrecht - z. B. beim Gewässerschutz - hinaus, wo zwar auch das kumulative Zusammenwirken erst die Rechtsgutsgefahr substanticll werden läßt, wo diese dann aber doch direkt auf die Tathandlung zurückgeführt werden kann. Außerdem bewirken solche »Kumulationsdelikte " erfahrungsgemäß eine Tcndenz zur Konzentration auf Bagatelldelikte und mithin ein Vollzugsdefizit bei schwerwiegenden Rechtsgutsverletzungen. ${ }^{39}$

6. Regelungstechnisch hätte man hier, folgerichtig und angemessener im Hinblick auf andere Strafrechtsbereiche, statt zur Figur des abstrakten Gefährdungsdeliktes eher zur materiell-rechtlichen Figur des »besonders schweren Falles " greifen müs-

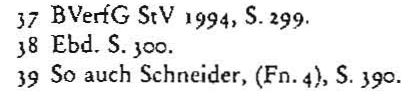


sen. Die Logik des abstrakten Gefährdungsdelikts wird dann aber doch noch durchbrochen, indem letztlich doch fallbezogen auf die konkrete Gefahr der Weitergabe abgestellt wird: Diese sci nämlich gering, wenn der Erwerb oder Besitz auf kleine Mengen zum gelegentlichen Eigenverbrauch beschränkt bleibe. Hier wird nun doch - und das ist die einzige Stelle in der Entscheidung, wo kriminologische Folgenforschung eine Rolle spielt - eine Folge der allzu rigorosen Anwendung des Prinzips abstrakter Gefährdungstatbestände reflektiert, z. B. die »unerwünschte Abdrängung in die Drogenszene und eine Solidarisierung mit ihr «. Es wird letztlich das bereits geltende strafprozeßrechtliche Korrektiv des $\$ 29$ Abs. 5 und des $\$ 3$ Ia BtMG beschworen, wobei letzterer eben schon das Absehen von Strafverfolgung, also die staatsanwaltschaftliche Einstellung ermöglicht, was wiederum längst nach $\$ \$$ i $53 \mathrm{ff}$. $\mathrm{StPO}$ möglich war und tatsächlich in Cannabis-Fällen weitläufig praktiziert wurde. $\$$ 3ra BtMG soll aber, und darin ist das BVerfG noch restriktiver als das geitende Recht, nur für Gelegenheitskonsumenten gelten und nicht bei "Frcmdgefährdung ", also z. B. nicht im Falle des Konsums in der Öffentlichkeit, vor Schulen und Kasernen. ${ }^{\text {to }}$

Genau hier ist die Passage der Entschcidung angesiedclt, welche zum cingangs erwähnten öffentlichen Mißverständnis geführt hat: Der Senat sieht in der Tat die faktische Ungleichbehandlung von Beschuldigten, die sich in der zwischen Regionen und Gerichtsbezirken extrem divergierenden Einstcllungspraxis gegcnüber Umgang mit geringer Menge manifestiert, z. B. 5,9\% aller solchen Verfahren in Baycrn, dagegen $79 \%$ in Berlin. ${ }^{41}$ Er fordert deshalb von den Justizverwaltungen der Länder, durch Verwaltungsvorschriften zu einer einheitlichen Einstellungspraxis der Staatsanwaltschaften zu gelangen. Dazu gehört die vage formulierte Forderung, daß hierzu Rechtstatsachenforschung zu betrciben sei..$^{2}$

Der Senat befaßt sich durchaus mit der grundrechtstheorctisch und kriminalpolitisch bedeutsamen Frage, ob nicht dafür eine materiell-rechtliche Regelung geboten sei, bleibt aber auch hier in der dogmatischen Qualität hinter der rechtswissenschaftlichen Dimension zurück:43 Lapidar wird crklärt, wcder das Übermaßverbor noch Art. 103 Abs. 2 GG seien durch die "prozessuale Lösung « tangiert, der Einzelne könne dem Gesetz mit hinreichender Dcutlichkeit cntnehmen, unter welchen Voraussetzungen er sich strafbar mache sowie welche Strafe ihm drohe. Dabei ist schon aus rein strafprozeßrechtlicher Sicht die dogmatische Haltbarkeit dieser Argumentation ebenso zweifelhaft wie die rechtliche und praktische Durchführbarkcit. ${ }^{44}$

Die unter bestimmten Umständen extrem hohe Strafbarkeit des Umgangs mit ciner "nicht geringen Mengc* wird umstandslos mit der abstrakt-generell größeren Rechtsgutsgefährdung legitimiert. Die Kritik des entsprechenden Vorlagebeschlusscs, die höchstrichterliche Festlegung der »nicht geringen Menge « von Cannabis bei 7,5 $\mathrm{g}$ verstoße gegen das Übermaßverbot, wird vom Senat mit dem Argument zurückgewiescn, diese Auslegung sei weder verbindlich noch endgülrig, und die Vorschrift könne bei Bedenken verfassungskonform ausgelegt werden. ${ }^{45}$ U.a. mit cntsprechender Begründung war übrigens der Frankfurter Vorlagebeschlu $\beta^{46}$ für unzulässig erklärt worden. Das Argument der fehlenden rechtlichen Bindung an die durch die BGH-Auslegung festgelegten Mengenbegriffe erscheint blauäugig, weil

\footnotetext{
40 Ebd. S. 3or.

4 I $V_{b} l$. den auch vom BVer $\{G$ zitierten Bericht der Bundesregierung BT-Drs. I I/4329, S. is ff.

42 BVerfG SiV 1994, S. 301 .

43 Zum neuesten Stand vgl. Nelles/Velten, (Fo. s), S. $366 \mathrm{ff}$.

44 Ebd.; ablehnend auch die abweichende Meinung von Sommer.

$45 \mathrm{BV} \cdot \mathrm{HG} \mathrm{StV}_{1994}, \mathrm{~S} .302$.

46 S. Fn. 3 .
} 
die BGH-Rechtsprechung faktisch eben doch starke normative Bindungswirkung entfaltet. Außerdem gerät das BVerfG auch hier in Widerspruch zu sich selbst, weil cs zum einen angesichts der unbestreitbaren Vagheit und Uferlosigkeit der Tatbestandsmerkmale des BtMG die gefestigte Auslegung des BGH als Garanten des Bestimmtheitsprinzips ansieht, die Fcstigkeit der Auslegung dann aber wiederum nicht wahrhaben will. Allerdings sollte diese Wendung den Untergerichten, Staatsanwaltschaften und Strafvertcidigem besonders ans Herz gelegt werden, verweist sie doch auf Aspekte des Prinzips richterlicher Unabhängigkcit, die aus dem Blick zu geraten drohen.

7. In Abschnitt $\mathrm{II}^{47}$ geht der Senat kurz und bündig mit dem - im Lübecker Vorlagebeschluß gebrauchten und in der Tat kaum triftigen - Argument ins Gericht, die Pönalisierung des Cannabis-Konsums zwinge den Konsumenten, auf andere, nicht dem BtMG unterliegende Rauschmittcl, wie z. B. Alkohl zurückzugreifen. Aufmerksam sollte man aber speichern, was der Senat hier betont: „Der Entschluß, sich durch den Mißbrauch solcher im Handel erhältlicher Rauschmittel selbst gesundheitlich zu schädigen, liegt vielmehr im Verantwortungsbereich der Konsumenten selbst. « Der Senat zieht hier selbstwidersprüchlich ein Argument heran, wclches er im zentralen Zusammenhang, nämlich der Frage der Fremdschädigung, so vehement zurückweist. Unerwähnt bleiben außerdem die - allerdings in den Vorlagebeschlüssen nicht argumentativ angcführten - mittelbaren Gesundheitsschäden durch Kriminalisierung und dissoziale Folgewirkungen derselben. ${ }^{48}$

8. Zurückgewiesen wird vom Senat weiter das Monitum der Ungleichbehandlung von Cannabis- und Alkohol-Konsumenten i.S. Art. 3 Abs. I GG (Abschnitt III). Verfassungsrechtlich ist kaum etwas dagegen cinzuwenden, daß dem Gesetzgeber ein Ermessensspielraum zugestanden wird, zu welchen Gesundheitsgefahren er sich durch gesetzliche Regelungen verhält und daß, solange es sachliche Differenzen im sozialen Umgang und in der Drogenwirkung gibt, der »allgemeine Gleichheitssatz nicht vorschreibt, alle BtM in gleicher Weise für den allgemeinen Verkehr freizugeben, weil andere gesundheitsgefährdende Stoffe zugclassen sind. « ${ }^{49}$ Jedoch erscheint die Begründung unzulänglich, mit welcher eine die Ungleichbehandlung rechtfertigende Ungleichartigkeit von Alkohol und Cannabis behauptet wird. Zum einen fehlt es an jeglicher ausdrücklichen bcgrifflichen Klärung, was eigentlich unter $\mathrm{BtM}$, Rausch, Genuß etc. verstanden wird. Die impliziten Begriffe werden zudem in widersprüchlicher Weise verwendet. So wird Cannabis als BtM definiert und daraus offenbar auf betäubende oder berauschende Wirkung geschlossen, während Nikotin kein $\mathrm{BtM}$ sei. Damit wird übergangen, daß - je nach Dosierung - Cannabis ein reines Genußmittel mit sehr milder Wirkung sein kann, wie es hier wohl unausgesprochen für Nikotin angenommen wird, welches ja dem Lebensmittel- und Bedarfsgegenstände-Gesetz (LBG) unterfällt. THC führt erst bei höherer Dosierung zu halluzinationsähnlichen Sinnesempfindungen und bei sehr hoher Dosierung zu Bewußtseinsstörungen. Überdosierungen mit Morbidität oder gar Mortalität sind nicht bekannt. Neueste Untersuchungen über Verhalten beim Autofahren unter Cannabis-Einfluß belegen beispielsweise, daß die psychologisch meßbaren Leistungen z. B. Reaktionsvermögen - in ihren absoluten Werten bei mäßigem Cannabis-Konsum nicht nachlassen, daß es lediglich Zeitverzögerungen im Entscheidungsverhalten gibr (z. B. übervorsichtiges Fahren). ${ }^{\circ}$ Langzeitwirkungen wie körperliche Abhängigkeit und zunehmende physiologische Zellschädigungen sind nicht bekannt.

47 BVerfG StV 1994, S. 302.

48 Ausführlich dazu das vom BVerfG zitierte Buch von Stephan Quensel: Drogenclend. Frankfurt 1982. 49 BVerfG StV 1994, S. 302.

so S. Fn. 28. 
Diesseits jeglicher Alkoholforschung und offenbar eher im Erfahrungsbereich des bürgerlichen Wein-Liebhabers angesiedelt ist die Behauptung des Senats, bei allem zugestandenen Mißbrauch dominiere in »allen Fällen ... eine Verwendung des Alkohols, dic nicht zu Rauschzuständen führt; seine berauschende Wirkung ist allgemein bekannt und wird durch soziale Kontrolle überwiegend vermicden. Demgegenüber steht beim Konsum von Cannabisprodukten typischerweise die Erzielung einer berauschenden Wirkung im Vordergrund . $^{\prime 1}$ Pharmakologisch, psycho-pharmakologisch, psychologisch und soziologisch steht außer Frage, daß Alkohol schon in geringen Mengen psychotrope Wirkung hat, welche je nach Dosierung, subjektiver Disposition und Erwartung ("Set ") und sozialer Konsumsituation ("Setting «) bis zu "Rausch« im Sinne des Alltagssprachgebrauchs, zu Bewußtseinsstörungen, Bewußtlosigkeit und spontanem Exitus führen können. Daß Alkohol auch in Medikamenten oder, wie der Senat feststellt, im religiösen Kult verwendet wird, widerlegt nicht die allscits bekannte und intendierte psychotrope Wirkung. Demgegenüber wird unterschlagen, daß, wenn Cannabis nicht illegal wäre, es gleichfalls vielfältigen anderen sozial-konstruktiven Gebrauchsformen zugeführt würde, insbesondere der medizinisch indizierten Verwendung. ${ }^{52}$ Das Argument, die kulturintegrierte Droge Alkohol könne auch aus faktischen Gründen nicht effektiv verboten werden, gilt im übrigen heute angesichts der zugestandenen hohen Verbreitung und faktisch leichten Beschaffbarkeit durchaus auch für Cannabis.

Schließlich wird noch dem Argument begegnet, die unterschiedslose strafrechtliche Behandlung von "weichen* und "harten "Drogen verstoße gegen Art. 3 Abs. I GG. Da eine solche Unterscheidung wegen der spezifischen Abhängigkeit der Wirkung von Drogenart und Dosierung, persönlicher Struktur und Erwartung, sozialem Umfeld und Konsum-Setting ohnehin problematisch ist, erscheint mir die Maßgabe des Senats folgerichtig und sinnvoll, insoweit die Gefährlichkcits-Abstufungen und damit die Schuld- und Strafbemessung der richterlichen Einzelfallbeurteilung zu überlassen.

\section{Die abweichenden Voten}

1. In ihrem abweichenden Votum kritisiert die Verfassungsrichterin Graßhof zutreffend den unreflektierten Umgang der Senatsmehrheit mit dem Instrument der abstrakten Gefährdungsdeliktc. Auch sie teilt jedoch die Vorgehensweise, in einer ersten Stufe der Prüfung nach Geeignetheit und Erforderlichkeit der Pönalisierung zu fragen und die Übermaßproblematik nur im Hinblick auf den Gesetzesvollzug zu prüfen (S. 2). Fragwürdig erscheint, daß sie bei dieser letzten Stufe lediglich »weniger rationale Maßstäbe von Zumutbarkeit und Angemessenheit « angewender wissen will, welche "in erster Linie der Korrektur offensichtlich unhaltbarer Ergebnisse" dienen. Von daher kommt sie zu dem Ergebnis, daß, wenn der Geserzgeber strafrechtliche Maßnahmen als Mittel zum Rechtsgüterschutz einsetzt, er dies nicht über das Übermaßverbot gleichsam wieder dementieren dürfe. Gerade bei der durch "Massenverstöße kumulierten Rechtsgutsbedrohung durch das Cannabis ist ihrer Meinung nach eine Relativierung hinsichtlich der Strafverfolgung nicht zu vertreten. Sie postuliert noch eindeutiger als die Senatsmehrheit das durch den massenhaften

SI BVerfG StV $1994, \mathrm{~S}_{303}$

52 So auch Schneider 1994 (Fn. 4), S. 391 ; vgl. zur medizinischen Indikavion: Grinspoon, Lester/Bakalar, James B.: Marihuana - Die verbotene Medizin, Frankfurt 1994. 
Cannabiskonsum gefährdete "Rechtsgut der Gestaltung des sozialen Zusammenlebens", sieht Konkretisierungen dieser Gefahr auch in den Straßenverkehrsrisiken (S. I 2). Weil jeder Verbraucher, auch der Gelegenheitskonsument, den Markt mittrage, müsse unterschiedslos verfolgt werden, wobei lediglich individuell wie sonst auch Unrecht und Schuld zu gewichten seien (S. I 8).

2. Geradezu radikal wirkt dagegen das abweichende Votum des Verfassungsrichters Sommer. Hier finden sich einige Argumente, wie sie auch hier vertreten werden. Insbesondere vertritt er, daß hinsichtlich Verbot, Strafandrohung und Strafverfolgung insgesamt der Maßstab des Verhältnismäßigkeitsprinzips einschließlich seiner Unterkriterien anzulegen sei, weil bereits die Strafandrohung wegen der massiven sozialethischen Unrechtszuschreibung ein Grundrechtseingriff sei (S. r). Gottlob, möchte man rufen, daß es diese klare und $\mathrm{m}$. E. richtige Position im obersten Verfassungsgericht (noch) gibt. Unter Verwendung der vom Senat selbst gefundenen reduzierten Schadenseinschätzung kommt Sommer auf rein normativem Wege zu dem Schluß, daß allenfalls Ordnungsunrecht noch ein adäquater Definitionsrahmen für den Umgang von Konsumenten mit Cannabis sein könne. Er sieht auch, daß dic Begründungen der Senatsmehrheit bzw. des Gesetzgebers zu immer abstrakteren Formen der Vorfeldverlagerung von Strafbarkeit führen (S. 6). Ihm entgeht allerdings der zentrale logische Fehler, daß das Gesetz nicht direkte Fremdgefährdung pönalisiert, sondern die unterstellte psychologische Motivierung zur Nachahmung. Zutreffend kritisiert er auch die Denkfigur der generellen Weitergabebereitschaft eines Konsumenten, der Drogen erwirbt oder besitzt (S. 7). Soweit Konsumenten dafür haftbar gemacht werden sollen, daß sie den Naclifrage-Anteil des Marktes konstituieren und es ein eventuelles Nachahmungsverhalten gibt, sieht er Art. I GG verletzt, indem sie »letztlich für vermutete schädliche Neigungen bzw. kriminelles Verhalten Dritter zur Verantwortung" gezogen werden (S.9). Im übrigen sieht er auch in den völkerrechtlichen Verpflichtungen zu Recht keinen Grund für eine Ablehnung der Entkriminalisierung (S. ro). Schließlich argumentiert er zutreffend mit Art. 103 Abs. 2 GG, daß - wenn schon die Pönalisierung beibehalten wird - eine materiellrecht liche Lösung der relativen Entkriminalisierung zu finden sei (S. I 3 ). Er begründet dies mit der Bedenklichkeit der inflationären symbolischen Instrumentalisierung des Strafrechts, welches seine Aussagekraft reduziere ( $S$. $1_{4}$ ). Im übrigen sei schon die Eröffnung eines Ermittlungsverfahrens eben ein Grundrechtseingriff.

\section{Resüme}

Zwei "Meilensteine" kennzeichnen den Cannabis-Beschluß des BVerfG - man könnte sie auch als Mühlsteine um den Hals des liberalen Tatstrafrechts bezeichnen. Den einen sehe ich in der schier grenzenlosen Ausdehnung der durch das BtMG geschützten Rechtsgüter. Diese ist insofern von allgemeiner Bedeutung, als die damit verbundene Entsubstantiierung und Entpersonalisierung des Rechtsgutsbegriffs auf andere Strafrechtsbereiche übergrcifen wird. Dem Staat sind insofern keine sachlichen Grenzen der Pönalisierung mehr gesetzt, substanzlose und immer abstraktere "soziale Rechtsgüter" können nunmehr bedenkenlos strafbewehrt werden. Am Ende könnte alles für strafbar erklärt werden, was dem Staat abträglich ist. Den anderen Mühlstein sehe ich in der ebenso grenzenlosen Entsubstantiierung und Symbolisierung des Strafrechts selbst. Durch die Entkoppelung von Strafandrohung und -verhängung bzw. -vollstreckung kommt es zu einer Verwischung der Grenzen 
zwischen Verwaltungs-, Ordnungs-, Polizei- und Strafrecht, zu einer Verharmlosung und Bagatellisierung des der Strafe strukturell innewohnenden besonderen und extremen sozialethischen Unwerturteils, zu einer Auflösung des ultima-ratio-Prinzips und damit des Gewichts des Strafübels im Rahmen der je notwendigen Abwägung. Auch damit ist der Weg zu einem immer hemmungsloseren Einsatz des Strafrechts weiter gebahnt.

Einc gravierende negative Bedeutung hinsichtlich unserer Rechtskultur sehe ich auch in dem nachlässigen Umgang mit den Kunst- und Sorgfaltsregeln justizieller und legislatorischer Methodik. Der Auftrag, die "materielle Wahrheit" des Regelungsbereiches im allgemcinen und des Lebenssachverhaltes im besonderen näherungsweise zu erarbeiten und in einer für andere verstehbarcn und nachvollziehbaren Weise darzustellen, erscheint mir nicht erfüllt. Hier ist lediglich eine oberflächliche "prozessuale Wahrheit« zum Zwecke der vordergründigen Entscheidungslegitimation hergestellt worden, die mit der Wirklichkeit des sozialen Problems wenig zu tun hat. 